\title{
A New Eigenfilter Based on Total Least Squares Error Criterion
}

\author{
Soo-Chang Pei, Fellow, IEEE and Chien-Cheng Tseng, Member, IEEE
}

\begin{abstract}
In this paper, a new eigenfilter based on total least squares error criterion is investigated. The filter coefficients are obtained from the elements of the eigenvector corresponding to minimum eigenvalue of a real, symmetric and positive definite matrix. Four features of new method are given below. First, the computation of filter coefficients of new eigenfilter is more numerically stable than that of the least-squares method whose solution is obtained by solving matrix inverse. Second, new eigenfilter does not need a reference frequency point for normalization as done in traditional eigenfilter. Third, the solution of the new eigenfilter is closer to the solution of the least-squares method than one of the conventional eigenfilter. Fourth, the proposed method is easy to incorporate with linear constraints and can be extended to design equiripple and two dimensional linear phase filters. Several design examples are used to illustrate the effectiveness of this new design approach.
\end{abstract}

Index Terms-Eigenfilter, least-squares design, total least-squares criterion.

\section{INTRODUCTION}

C ONVENTIONALLY, we often use the well-known McClellan-Parks-Rabiner (MPR) computer program and standard linear programming technique to design linear phase finite impulse respones (FIR) filters according to the Chebyshev criterion which minimizes the maximum error in frequency response [1], [2]. The minimax designs usually give the designers a smallest length filter for a given specification. However, it is difficult for the MPR algorithm to incorporate both a time- and frequency-domain constraint. And, linear programming technique needs a large memory space and considerable computation time. Thus, a number of researchers have considered linear phase FIR filter design using least-squares optimality criterion.

From literature survey, two well-documented least squares approaches for FIR filter designs are inverse matrix (IM) method and eigen-approach. References [3]-[8] are examples of publications which include descriptions of using two methods to design various filters. The IM methods are based on solving a set of linear simultaneous equations by matrix inversion [3], [4], and the eigen-approaches are based on the computation of an eigenvector of an appropriate real, symmetric, and positive-definite matrix [5]-[8]. Compared with minimax design, the advantage

Manuscript received February 24, 2000; revised November 15, 2000. This paper was recommended by Associate Editor P. P. Vaidyanathan.

S.-C. Pei is with the Department of Electrical Engineering, National Taiwan University, Taipei, Taiwan, R.O.C.

C.-C. Tseng is with the Department of Computer and Communication Engineering, National Kaohsiung First University of Science and Technology, Kaohsiung, Taiwan, R.O.C.

Publisher Item Identifier S 1057-7122(01)04289-1. of least squares design is that it is easy to add constraints and it requires simple computation. So far, least squares approach has been widely used to design various filters in multirate applications and image processing [9], [10].

The purpose of this paper is to design linear phase FIR filters using total least squares (TLS) error criterion which has been successfully used to solve many engineering problems such as acoustic radiation problem, adaptive filtering, beamformer and harmonic retrieval etc., [11]-[13]. The filter coefficients based on TLS criterion are obtained from the elements of the eigenvector corresponding to minimum eigenvalue of a real, symmetric and positive definite matrix. Due to this, the total least squares filter design is referred to as the new eigenfilter approach. The main difference between conventional and new eigenfilters is that conventional method needs to specify the reference point in frequency domain, but new approach does not require this. Moreover, three unique features of new eigenfilter are as follows. First, the computation of filter coefficients of new eigenfilter is more numerically stable than the least-squares method whose solution is obtained by solving IM. Second, the solution of the new eigenfilter is closer to the solution of the least-squares method than one of the conventional eigenfilter. Third, it is easy for the new eigenfilter to incorporate time domain constraints and other linear constraints as for the traditional eigenfilter. Moreover, the new eigenfilter can be extended to design equiripple and two dimensional linear phase filters.

The paper is organized as follows. In Section II, the linear phase FIR filter designs using conventional IM method and eigen-approach are briefly reviewed. In Section III, a new eigenfilter based on total least squares error criterion is developed. In Section IV, we extend the new eigenfilter approach to design FIR filters in conjunction with general linear constraints. The notch filter with controlled null width is presented to show the effectiveness of our method. In Section V, we use iterative weighted total least squares method to design equiripple linear phase FIR filters. Finally, the new eigen-approach is modified to design two dimensional quadrantally symmetric FIR filters and concluding remarks are made.

\section{Conventional Least SQuares Filter Design}

\section{A. Problem Statement}

A causal $N$-th order FIR filter can be represented as

$$
H(z)=\sum_{n=0}^{N} h(n) z^{-n} .
$$


This filter is said to have linear phase if phase response is linear in frequency. Depending on whether $N$ is even or odd, and whether $h(n)$ is symmetric or antisymmetric, we obtain four types of real coefficient linear phase filters [2]. The magnitude responses of these four types of filters can be expressed as

$$
A(\omega)=\sum_{n=1}^{M} a_{n} \operatorname{trig}(\omega, n)
$$

where $\operatorname{trig}(\omega, n)$ is an appropriate trigonometrical function. The coefficient $a_{n}$ is related to the impulse response of the filter, whereas $M$ is a function of the filter order $N$. Defining the column vector

$$
\mathbf{a}=\left[\begin{array}{llll}
a_{1} & a_{2} & \ldots & a_{M}
\end{array}\right]^{t}
$$

and

$$
\mathbf{c}(\omega)=[\operatorname{trig}(\omega, 1) \operatorname{trig}(\omega, 2) \quad \ldots \quad \operatorname{trig}(\omega, M)]^{t}
$$

then we rewrite (2) as

$$
A(\omega)=\mathbf{a}^{t} \mathbf{c}(\omega)=\mathbf{c}^{t}(\omega) \mathbf{a} .
$$

The notation $t$ denotes the vector or matrix transpose. Now, the problem is how we can find a such that the magnitude response $A(\omega)$ in (5) fits the desired magnitude response $D(\omega)$ as well as possible. Various least squares error measures will be used through this paper.

\section{B. Conventional Least Squares Filter Design}

The conventional least squares approach corresponds to minimizing the following objective function [Tufts and Francis, 1970]

$$
\begin{aligned}
J_{t}(\mathbf{a}) & =\int_{\omega \in R}(D(\omega)-A(\omega))^{2} d \omega \\
& =\mathbf{a}^{t} \mathbf{Q}_{\mathbf{t}} \mathbf{a}-2 \mathbf{p}^{t} \mathbf{a}+d
\end{aligned}
$$

where $R$ is the region $0 \leq \omega \leq \pi$, but excluding the transition band. The matrix $\mathbf{Q}_{\mathbf{t}}$, vector $\mathbf{p}$, and scalar $d$ are

$$
\begin{aligned}
\mathbf{Q}_{\mathbf{t}} & =\int_{\omega \in R} \mathbf{c}(\omega) \mathbf{c}^{t}(\omega) d \omega \\
\mathbf{p} & =\int_{\omega \in R} D(\omega) \mathbf{c}(\omega) d \omega \\
d & =\int_{\omega \in R} D^{2}(\omega) d \omega .
\end{aligned}
$$

Because $J_{t}(\mathbf{a})$ is a quadratic function of $\mathbf{a}$, the optimal value $\mathbf{a}_{t}$ that minimizes $J_{t}(\mathbf{a})$ must satisfy $\nabla \mathbf{J}_{t}\left(\mathbf{a}_{t}\right)=0$, where $\nabla \mathbf{J}_{t}(\mathbf{a})$ is the multivariable derivative of $\mathbf{J}_{t}(\mathbf{a})$. From (6)

$$
\nabla \mathbf{J}_{t}(\mathbf{a})=2 \mathbf{Q}_{t} \mathbf{a}-2 \mathbf{p} .
$$

Therefore, the optimal solution

$$
\mathbf{a}_{t}=\mathbf{Q}_{t}^{-1} \mathbf{p} .
$$

The actual value of $\mathbf{a}_{t}$ can be obtained by matrix inversion or by solving simultaneous linear equations

$$
\mathbf{Q}_{t} \mathbf{a}_{t}=\mathbf{p} .
$$

Because $\mathbf{Q}_{t}$ is a positive-definite, real, and symmetric matrix, the simultaneous linear equations can be solved by a computationally efficient method, like Cholesky decomposition [16]. Several interesting design examples of such a least squares FIR filters can be found in [3].

\section{Conventional Eigenfilter Design}

In the following, we will design linear phase FIR filter using another least squares error measure. The solution in this case is the eigenvector corresponding to the minimum eigenvalue of a real, symmetric, and positive definite matrix so that it is often referred to as eigenfilter approach in the literature. So far, eigenfilter approach has been used to design various types of filters such as lowpass filters, differentiators and Hilbert transformers [5]-[7]. The design procedure of these eigenfilters can be summarized a unified formulation as follows.

Step 1: Choose the reference point $\omega_{0}$ satisfying $D\left(\omega_{0}\right) \neq$ 0 . Some consideration of this choice can be found in [5]-[7].

Step 2: Make magnitude response $A(\omega)$ approximate the specification $\left(D(\omega) / D\left(\omega_{0}\right)\right) A\left(\omega_{0}\right)$. To achieve this approximation, we find the optimal solution a by minimizing the following quadratic error measure

$$
J_{e}(\mathbf{a})=\int_{\omega \in R}\left|\frac{D(\omega)}{D\left(\omega_{0}\right)} A\left(\omega_{0}\right)-A(\omega)\right|^{2} d \omega .
$$

Using (5), we can rewrite $J_{e}(\mathbf{a})$ as

$$
\begin{aligned}
J_{e}(\mathbf{a}) & =\int_{\omega \in R}\left|\frac{D(\omega)}{D\left(\omega_{0}\right)} \mathbf{a}^{t} \mathbf{c}\left(\omega_{0}\right)-\mathbf{a}^{t} \mathbf{c}(\omega)\right|^{2} d \omega \\
& =\mathbf{a}^{t} \mathbf{Q}_{e} \mathbf{a}
\end{aligned}
$$

where matrix

$$
\begin{aligned}
\mathbf{Q}_{e}= & \int_{\omega \in R}\left(\frac{D(\omega)}{D\left(\omega_{0}\right)} \mathbf{c}\left(\omega_{0}\right)-\mathbf{c}(\omega)\right) \\
& \cdot\left(\frac{D(\omega)}{D\left(\omega_{0}\right)} \mathbf{c}\left(\omega_{0}\right)-\mathbf{c}(\omega)\right)^{t} d \omega .
\end{aligned}
$$

Note that $\mathbf{Q}_{e}$ is a real, symmetric and positive-definite matrix. Obviously, the minimum of $J_{e}(\mathbf{a})$ occurs at $\mathbf{a}=\mathbf{0}$. To avoid trivial solutions, the constraint $\mathbf{a}^{t} \mathbf{a}=1$ is added. Under this condition, the solution vector $\mathbf{a}_{e}$ is simply the eigenvector of $\mathbf{Q}_{e}$ corresponding to its minimum eigenvalue in view of the well-known Rayleigh's principle [14].

Step 3: Since we have made $A(\omega)$ approximate $\left(D(\omega) / D\left(\omega_{0}\right)\right) A\left(\omega_{0}\right)$, we should scale the solution $\mathbf{a}_{e}$ properly to satisfy $\mathbf{a}_{e}^{t} \mathbf{c}\left(\omega_{0}\right)=D\left(\omega_{0}\right)$ [5]-[7]. Thus, the final solution is given by

$$
\mathbf{a}_{e f}=\frac{D\left(\omega_{0}\right)}{\mathbf{a}_{e}^{t} \mathbf{c}\left(\omega_{0}\right)} \mathbf{a}_{e} .
$$

For eigenfilter design, we are interested in only the minimum eigenvector of a symmetric matrix, this computation can be performed efficiently by the conjugate gradient method [15], or iterative power method [16]. 


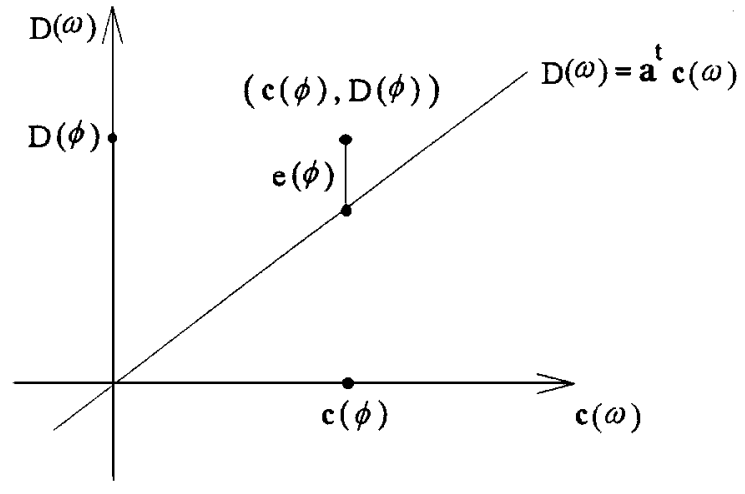

(a)

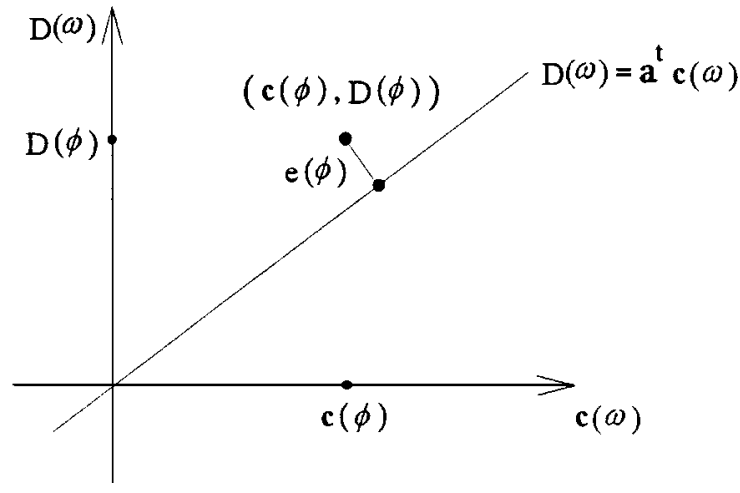

(b)

Fig. 1. Geometric interpretation of two error measures at frequency $\omega=\phi$ (a) Type 1 error. (b) Type 2 error.

\section{A NeW Eigenfilter BASEd ON TOtal LEASt SQuARES ERROR CRITERION}

\section{A. A New Eigenfilter Design}

The well-known linear phase filter design problem is to find a filter weight a such that the desired magnitude response $D(\omega)$ is equal to the actual magnitude response $\mathbf{a}^{t} \mathbf{c}(\omega)$ of the filter, i.e.,

$$
D(\omega)=\mathbf{a}^{t} \mathbf{c}(\omega)
$$

for each $\omega \in[0, \pi)$. In the space $\Re$ generated by $\mathbf{c}(\omega)$ and $D(\omega)$, it is clear that the expression $D(\omega)=\mathbf{a}^{t} \mathbf{c}(\omega)$ denotes a hyperplane. For a given frequency $\phi$, the $(\mathbf{c}(\phi), D(\phi))$ denotes a point in $\Re$. Now, the filter design problem can be restated as "we want the point $(\mathbf{c}(\phi), D(\phi))$ to fall on the hyperplane $D(\omega)=\mathbf{a}^{t} \mathbf{c}(\omega)$ for all $\phi \in[0, \pi)$." When the point $(\mathbf{c}(\phi), D(\phi))$ does not fall on the plane $D(\omega)=\mathbf{a}^{t} \mathbf{c}(\omega)$, the error between them can be measured in several ways. Two typical ones with geometric interpretation are shown in Fig. 1. One error (type 1) is given by

$$
e(\phi)=\left|D(\phi)-\mathbf{a}^{t} \mathbf{c}(\phi)\right|
$$

the other (type 2) is given by

$$
e(\phi)=\frac{\left|D(\phi)-\mathbf{a}^{t} \mathbf{c}(\phi)\right|}{\sqrt{\mathbf{a}^{t} \mathbf{a}+1}} .
$$

The least squares filter design problem means that the optimal filter weight $\mathbf{a}$ is obtained by minimizing the squared errors

$$
J(\mathbf{a})=\int_{\omega \in R}|e(\omega)|^{2} d \omega
$$

where $R$ is the region $0 \leq \omega \leq \pi$, but excluding the transition band. Now, two types of least squares errors will be investigated in detail. Substituting (16) into (18), we obtain

$$
J(\mathbf{a})=\int_{\omega \in R}\left(D(\omega)-\mathbf{a}^{t} \mathbf{c}(\omega)\right)^{2} d \omega
$$

which is same as the (6). Thus, this least squares error measure is equal to the conventional one. Next, substituting (17) into (18), we have

$$
\begin{aligned}
J(\mathbf{a}) & =\int_{\omega \in R} \frac{\left(D(\omega)-\mathbf{a}^{t} \mathbf{c}(\omega)\right)^{2}}{\mathbf{a}^{t} \mathbf{a}+1} d \omega \\
& =\frac{\hat{\mathbf{a}}^{t} \mathbf{Q}_{n} \hat{\mathbf{a}}}{\hat{\mathbf{a}}^{t} \hat{\mathbf{a}}}
\end{aligned}
$$

where $\hat{\mathbf{a}}$ and $\mathbf{Q}_{n}$ are given by

$$
\begin{aligned}
\hat{\mathbf{a}} & =\left[\begin{array}{ll}
\mathbf{a}^{t} & -1
\end{array}\right]^{t} \\
\hat{\mathbf{c}}(\omega) & =\left[\begin{array}{ll}
\mathbf{c}(\omega)^{t} & D(\omega)
\end{array}\right]^{t} \\
\mathbf{Q}_{n} & =\int_{\omega \in R} \hat{\mathbf{c}}(\omega) \hat{\mathbf{c}}(\omega)^{t} d \omega .
\end{aligned}
$$

Based on Rayleigh's principle, the minimum of $J(\mathbf{a})$ occurs at the eigenvector of the matrix $\mathbf{Q}_{n}$ corresponding to the minimum eigenvalue. Note that the $\mathbf{Q}_{n}$ is also a real, symmetric and positive definite matrix. Since the solution vector $\mathbf{a}$ is simply the minimum eigenvector of matrix $\mathbf{Q}_{n}$, we call this least squares design as new eigenfilter design. In [11], the least squares error of type 2 is named as total least squares error which have been successfully used to solve many engineering problems such as acoustic radiation problem, beamformer, structural identification and harmonic retrieval etc. We claim that the new eigenfilter is optimal in total least squares error sense. Now, we summarize the design procedure of new eigenfilter as follows.

Step 1: Compute the matrix $\mathbf{Q}_{n}$.

Step 2: Calculate the minimum eigenvector $\hat{\mathbf{a}}_{o}$ of the matrix $\mathbf{Q}_{n}$.

Step 3: Normalize the solution vector $\hat{\mathbf{a}}_{o}$ to the form $\left[\begin{array}{ll}\mathbf{a}_{o}^{t} & -1\end{array}\right]^{t}$. The final desired solution $\mathbf{a}_{n}$ is equal to $\mathbf{a}_{o}$.

Three main differences between conventional eigenfilter and new eigenfilter are listed below. First, conventional eigenfilter needs to specify the reference point, but new eigenfilter does not require this choice. Second, the size of the matrix $\mathbf{Q}_{e}$ of conventional eigenfilter is $M \times M$, but the size of the matrix $\mathbf{Q}_{n}$ of new eigenfilter is $(M+1) \times(M+1)$. Third, the normalization of conventional eigenfilter is to scale minimum eigenvector $\mathbf{a}_{e}$ to satisfy $\mathbf{a}_{e}^{t} \mathbf{c}\left(\omega_{0}\right)=D\left(\omega_{0}\right)$, but the normalization of new eigenfilter is to scale minimum eigenvector vector $\hat{\mathbf{a}}_{o}$ to the form $\left[\begin{array}{ll}\mathbf{a}_{o}^{t} & -1\end{array}\right]^{t}$. 


\section{B. A Unified Design Procedure}

In the following, we first study the relation between $\mathbf{Q}_{e}, \mathbf{Q}_{n}$ and $\mathbf{Q}_{t}$. Then, we develop a unified design procedure to obtain the solutions of three least squares approaches at the same time.

Fact 1: The relation between the matrix $\mathbf{Q}_{t}$ and the matrix $\mathbf{Q}_{e}$ is given by:

$$
\begin{aligned}
\mathbf{Q}_{e}= & \frac{d}{D\left(\omega_{0}\right)^{2}} \mathbf{c}\left(\omega_{0}\right) \mathbf{c}^{t}\left(\omega_{0}\right)-\frac{1}{D\left(\omega_{0}\right)} \\
& \cdot\left[\mathbf{c}\left(\omega_{0}\right) \mathbf{p}^{t}+\mathbf{p} \mathbf{c}^{t}\left(\omega_{0}\right)\right]+\mathbf{Q}_{t} .
\end{aligned}
$$

Proof: From (13), we have

$$
\begin{aligned}
\mathbf{Q}_{e}= & \frac{1}{D\left(\omega_{0}\right)^{2}} \mathbf{c}\left(\omega_{0}\right) \mathbf{c}^{t}\left(\omega_{0}\right) \int_{\omega \in R} D(\omega)^{2} d \omega \\
& -\frac{1}{D\left(\omega_{0}\right)} \mathbf{c}\left(\omega_{0}\right) \int_{\omega \in R} D(\omega) \mathbf{c}^{t}(\omega) d \omega \\
& -\frac{1}{D\left(\omega_{0}\right)} \int_{\omega \in R} D(\omega) \mathbf{c}(\omega) d \omega \mathbf{c}^{t}\left(\omega_{0}\right) \\
& +\int_{\omega \in R} \mathbf{c}(\omega) \mathbf{c}^{t}(\omega) d \omega .
\end{aligned}
$$

Substituting (7) into (22), we obtain (21). The proof is completed.

Fact 2: The relation between the matrix $\mathbf{Q}_{t}$ and the matrix $\mathbf{Q}_{n}$ is given by

$$
\mathbf{Q}_{n}=\left[\begin{array}{ll}
\mathbf{Q}_{t} & \mathbf{p} \\
\mathbf{p}^{t} & d
\end{array}\right]
$$

Proof: From (20), we have

$$
\begin{aligned}
\mathbf{Q}_{n} & =\int_{\omega \in R} \hat{\mathbf{c}}(\omega) \hat{\mathbf{c}}(\omega)^{t} d \omega \\
& =\left[\begin{array}{ll}
\int_{\omega \in R} \mathbf{c}(\omega) \mathbf{c}(\omega)^{t} d \omega & \int_{\omega \in R} \mathbf{c}(\omega) D(\omega) d \omega \\
\int_{\omega \in R} D(\omega) \mathbf{c}(\omega)^{t} d \omega & \int_{\omega \in R} D(\omega)^{2} d \omega
\end{array}\right] .
\end{aligned}
$$

Substituting (7) into (24), we obtain (23). The proof is completed.

Although the above two facts are not concerned with the performance of designed filters, they provide us a way to program three least squares methods in a unified procedure as follows.

Step 1: Calculate matrix $\mathbf{Q}_{t}$. The elements of $\mathbf{Q}_{t}$ are given by

$q_{t}(i, j)=\int_{R} \operatorname{trig}(\omega, i) \operatorname{trig}(\omega, j) d \omega, \quad 1 \leq i, j \leq M$.

Step 2: Compute vector $\mathbf{p}$ whose elements can be written as

$$
p(i)=\int_{R} \operatorname{trig}(\omega, i) D(\omega) d \omega, \quad 1 \leq i \leq M .
$$

Step 3: Find the solution of conventional least squares filter design, i.e., $\mathbf{a}_{t}=\mathbf{Q}_{t}^{-1} \mathbf{p}$.

Step 4: Use fact 1 to calculate matrix $\mathbf{Q}_{e}$.

Step 5: Find the minimum eigenvector $\mathbf{a}_{e}$ of matrix $\mathbf{Q}_{e}$. And, the solution of eigenfilter is given by $\mathbf{a}_{e f}=$ $\left(D\left(\omega_{0}\right) / \mathbf{a}_{e}^{t} \mathbf{c}\left(\omega_{0}\right)\right) \mathbf{a}_{e}$.
Step 6: Use fact 2 to calculate matrix $\mathbf{Q}_{n}$.

Step 7: Find the minimum eigenvector $\hat{\mathbf{a}}_{o}$ of matrix $\mathbf{Q}_{n}$. And, normalize the solution vector $\hat{\mathbf{a}}_{o}$ to the form $\left[\begin{array}{ll}\mathbf{a}_{o}^{t} & -1\end{array}\right]^{t}$. The final solution $\mathbf{a}_{n}$ is written as $\mathbf{a}_{o}$.

\section{Design Example}

In the example, we will compare the performance of three least squares methods. This example is performed with the MATLAB Language in an IBM PC compatible computer by using the unified design procedure.

Example 1: Lowpass Filter Design: Consider the problem of designing a lowpass filter with the following desired amplitude response

$$
D(\omega)= \begin{cases}1, & 0 \leq \omega \leq \omega_{p} \\ 0, & \omega_{s} \leq \omega \leq \pi \\ \text { don't care, } & \omega_{p}<\omega<\omega_{s} .\end{cases}
$$

There are four cases of FIR filters with exactly linear phase, but only two of these could be applied to design lowpass filters, that is, case 1 and case 2 [2]. Here, we only consider case 1 filter whose elements of matrix $\mathbf{Q}_{t}$ and $\mathbf{p}$ are given by

$$
\begin{aligned}
q_{t}(i, j)= & \int_{R} \cos ((i-1) \omega) \cos ((j-1) \omega) d \omega, \\
& \quad 1 \leq i, j \leq M \\
p(i)= & \int_{R} \cos ((i-1) \omega) D(\omega) d \omega, \quad 1 \leq i \leq M
\end{aligned}
$$

where the region $R=\left[0, \omega_{p}\right] \cup\left[\omega_{s}, \pi\right]$. Now, two experiments are made. The reference point $\omega_{0}$ chosen in the conventional eigenfilter is $\omega_{p} / 2$. The least squares solution is obtained using "inv.m" of MATLAB, and eigenfilters are designed using "eig.m." First, Fig. 2(a) shows the magnitude responses of three least squares approaches with order $N=32, \omega_{p}=0.2 \pi$, and $\omega_{s}=0.3 \pi$. From this result, it is clear that the specification are well satisfied for three least squares methods. However, the stopband attenuation of conventional eigenfilter is slightly worse than the other two methods, because the amplitude response at the reference frequency $\omega_{0}$ must be satisfied exactly for the conventional eigenfilter design. Moreover, the distances between the solutions of three methods are listed as follows:

$$
\begin{aligned}
\left|\mathbf{a}_{e f}-\mathbf{a}_{t}\right| & =0.005109 \\
\left|\mathbf{a}_{n}-\mathbf{a}_{t}\right| & =0.000267 .
\end{aligned}
$$

Thus, the solution of the new eigenfilter is closer to the solution of the conventional least squares method than the one of the conventional eigenfilter. Second, we consider the design of high order filters with large transition band. Fig. 2(b) shows the magnitude responses of three approaches with order $N=148$, $\omega_{p}=0.25 \pi$, and $\omega_{s}=0.4 \pi$. It is clear that the design results of least squares method is very bad in the stopband, but two eigenfilter approaches still work well. This is due to ill conditioning of matrix $\mathbf{Q}_{t}$ whose determinant is $6 \times 10^{-54}$. Thus, the computation of filter coefficients of new eigenfilter is more numerically 


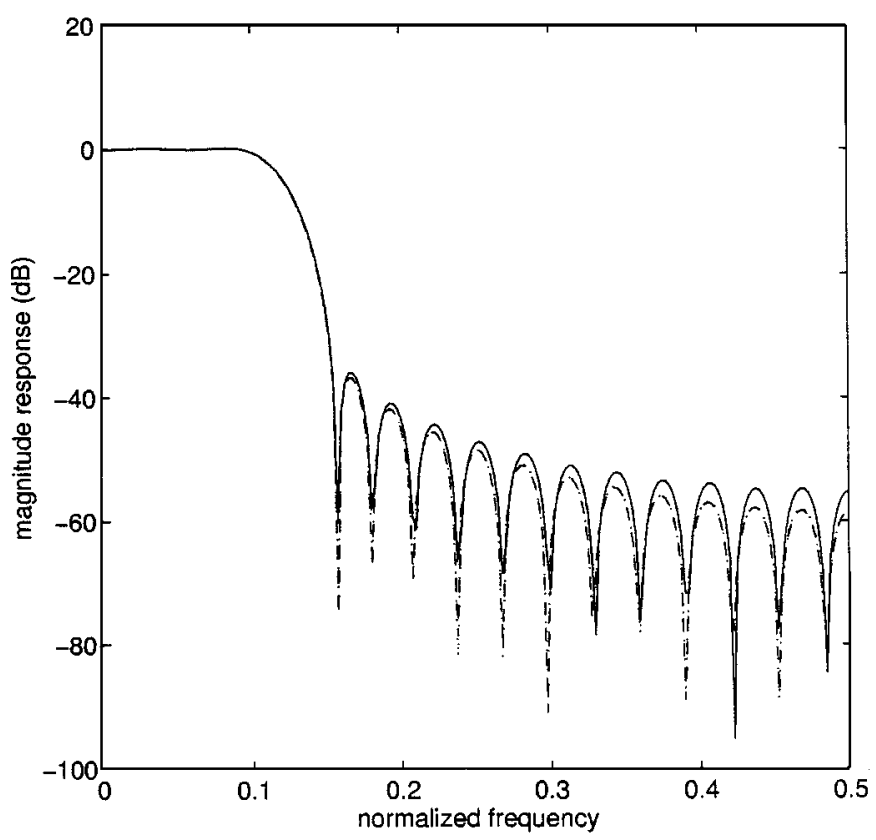

(a)



(b)

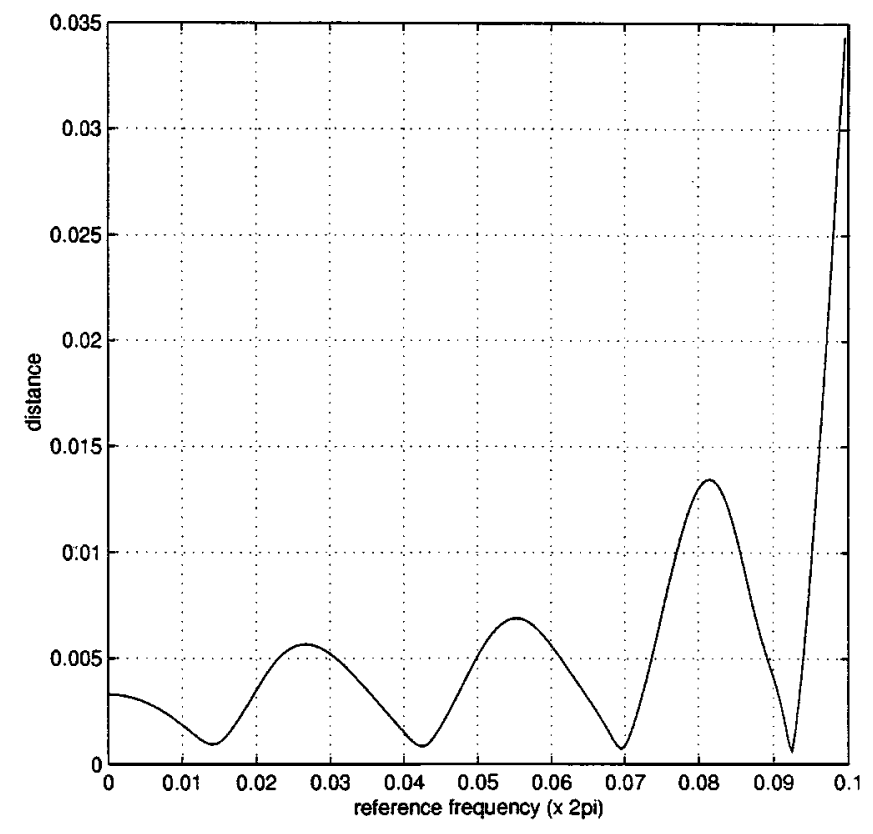

(c)

Fig. 2. (a) The magnitude response of a lowpass filter with $\omega_{p}=0.2 \pi, \omega_{s}=0.3 \pi$, and $N=32$. The dashed line and dotted line are almost overlap. conventional least squares design (dashed line), conventional eigenfilter design (solid line), new eigenfilter design (dotted line). (b) The magnitude response of a lowpass filter with $\omega_{p}=0.25 \pi, \omega_{s}=0.4 \pi$, and $N=148$. The solid line and dotted line almost overlap. The conventional least squares design is represented by the dashed line, the conventional eigenfilter design by the solid line, and the new eigenfilter design by the dotted line. (c) The distance $\left|\mathbf{a}_{e f}-\mathbf{a}_{t}\right|$ for various reference frequency points $\omega_{o}$ in the range $\left[0, \omega_{p}\right]$. The specification is chosen as $\omega_{p}=0.2 \pi, \omega_{s}=0.3 \pi$, and $N=32$.

stable than that of the least-squares method whose solution is obtained by solving matrix inverse.

Finally, a remark is made. Because the solution $\mathbf{a}_{e f}$ of conventional eigenfilter depends on the choice of reference frequency point $\omega_{o}$, it is useful to investigate the relation between distance $\left|\mathbf{a}_{e f}-\mathbf{a}_{t}\right|$ and reference frequency $\omega_{o}$. Fig. 2(c) shows the distance $\left|\mathbf{a}_{e f}-\mathbf{a}_{t}\right|$ for various reference frequencies $\omega_{o}$ in the range $\left[0, \omega_{p}\right]$ when the specification is chosen as $N=32$, $\omega_{p}=0.2 \pi$ and $\omega_{s}=0.3 \pi$. From the result, we see that the distance $\left|\mathbf{a}_{e f}-\mathbf{a}_{t}\right|$ has minimum value 0.000572 when frequency $\omega_{0}=0.186 \pi$. However, the distance $\left|\mathbf{a}_{n}-\mathbf{a}_{t}\right|$ is equal to 0.000267 , so the distance $\left|\mathbf{a}_{e f}-\mathbf{a}_{t}\right|$ is always greater than $\left|\mathbf{a}_{n}-\mathbf{a}_{t}\right|$ for all reference frequencies. This means that the solution of the new eigenfilter is closer to the solution of the conventional least squares method than the one of the conventional eigenfilter for all choice of reference frequency $\omega_{0}$. 


\section{New Eigenfilter Design with Linear Constraint}

The main advantage of the least squares approach is that various time and frequency constraints can be incorporated. In the linear case, the general form of the constraints can be stated as

$$
\mathrm{Ea}=\mathrm{k}
$$

where $\mathbf{E}$ is an $L \times M$ matrix and $\mathbf{k}$ is an $L \times 1$ vector. Note that $L$ is the number of the linear constraints which is usually smaller than the number of coefficients $M$. Moreover, we assume $\mathbf{E}$ is a full rank matrix in order to avoid redundant constraints. For the conventional least squares filter design, the closed-form solution can be obtained by using the well-known Lagrange multiplier method [17]. Moreover, the procedure to find the solution of the conventional constrained eigenfilter design is slightly complicated, the detail can be found in [5], [18]. As to the new eigenfilter design which is our main focus, the design problem becomes

Minimize

$$
\frac{\hat{\mathbf{a}}^{t} \mathbf{Q}_{n} \hat{\mathbf{a}}}{\hat{\mathbf{a}}^{t} \hat{\mathbf{a}}}
$$

Subject to

$$
\mathbf{E a}=\mathbf{k}
$$

where $\hat{\mathbf{a}}^{t}=\left[\mathbf{a}^{t}-1\right]$. The basic idea of solving this problem is to rewrite the constraint $\mathbf{E a}=\mathbf{k}$ as the following form:

$$
\hat{\mathbf{E}} \hat{\mathbf{a}}=\mathbf{0}
$$

where $\hat{\mathbf{E}}=[\mathbf{E} \mathbf{k}]$. Then, the problem is reduced to

\section{Minimize}

Subject to

$$
\frac{\hat{\mathbf{a}}^{t} \mathbf{Q}_{n} \hat{\mathbf{a}}}{\hat{\mathbf{a}}^{t} \hat{\mathbf{a}}}
$$

$$
\hat{\mathbf{E}} \hat{\mathbf{a}}=\mathbf{0} .
$$

The key step of our method is that "all the vector â satisfying constraint $\hat{\mathbf{E}} \hat{\mathbf{a}}=\mathbf{0}$ can be expressed as $\hat{\mathbf{a}}=\mathbf{B w}$, where the columns of $\mathbf{B}$ form an orthonormal basis of the null space of matrix $\hat{\mathbf{E}}$." Based on full rank assumption of $\mathbf{E}$, we have that $\mathbf{B}$ is a $(M+1) \times(M+1-L)$ matrix and $\mathbf{w}$ is a $(M+1-L) \times$ 1 vector. Due to orthonormal condition, we obtain $\mathbf{B}^{t} \mathbf{B}=\mathbf{I}$, where $\mathbf{I}$ is a $(M+1-L) \times(M+1-L)$ identity matrix. Thus, the problem described in (28) can be simplified as

\section{Minimize}

$$
\frac{\mathbf{w}^{t} \mathbf{B}^{t} \mathbf{Q}_{n} \mathbf{B w}}{\mathbf{w}^{t} \mathbf{w}}
$$

which is an unconstrained optimization problem. Hence, the optimal solution $\mathbf{w}_{O}$ of this simplified problem is the minimum eigenvector of matrix $\mathbf{B}^{t} \mathbf{Q}_{n} \mathbf{B}$. Finally, the desired optimal solution $\hat{\mathbf{a}}_{o}$ is equal to $\mathbf{B w}_{o}$. Now, the remaining problem is how to find the orthonormal basis of null space of matrix $\hat{\mathbf{E}}$. Any of the following two facts will help us to solve it [16].

Fact 3: Let singular value decomposition (SVD) [14] of matrix $\hat{\mathbf{E}}$ be

$$
\hat{\mathbf{E}}=\mathbf{U} \boldsymbol{\Sigma} \mathbf{V}^{t}
$$

where unitary matrices $\mathbf{U}, \mathbf{V}$ and diagonal matrix $\mathbf{\Sigma}$ are

$$
\begin{aligned}
\mathbf{U} & =\left[\mathbf{u}_{1}, \mathbf{u}_{2}, \ldots, \mathbf{u}_{L}\right] \\
\mathbf{V} & =\left[\mathbf{v}_{1}, \mathbf{v}_{2}, \ldots, \mathbf{v}_{M+1}\right] \\
\mathbf{\Sigma} & =\operatorname{diag}\left(\sigma_{1}, \sigma_{2}, \ldots, \sigma_{L}\right) .
\end{aligned}
$$

Then, the null space of matrix $\hat{\mathbf{E}}=\operatorname{span}\left\{\mathbf{v}_{L+1}, \mathbf{v}_{L+2}, \ldots, \mathbf{v}_{M+1}\right\}$.

Fact 4: Let QR decomposition [14] of matrix $\hat{\mathbf{E}}^{t}$ be

$$
\hat{\mathbf{E}}^{t}=\mathbf{Q}\left[\begin{array}{c}
\mathbf{R} \\
\mathbf{0}
\end{array}\right]
$$

where

unitary matrix $\mathbf{Q}\left[\mathbf{v}_{1}, \mathbf{v}_{2}, \ldots, \mathbf{v}_{M+1}\right]$ with size $(M+1) \times$ $(M+1)$;

$\mathbf{R} \quad$ upper triangular matrix with size $L \times L$;

0 a zero matrix with size $(M+1-L) \times L$.

Then, the null space of $\hat{\mathbf{E}}=\operatorname{span}\left\{\mathbf{v}_{L+1}, \mathbf{v}_{L+2}, \ldots, \mathbf{v}_{M+1}\right\}$.

Finally, we summarize the entire procedure of the proposed method as follows:

Step 1: Use SVD or QR decomposition to find the orthonormal basis of null space of matrix $\hat{\mathbf{E}}$ and construct the matrix $\mathbf{B}$.

Step 2: Find the minimum eigenvector $\mathbf{w}_{o}$ of matrix $\mathrm{B}^{t} \mathrm{Q}_{n} \mathrm{~B}$.

Step 3: Calculate the optimal solution $\hat{\mathbf{a}}_{o}=\mathbf{B w}_{o}$.

Step 4: Normalize the solution vector $\hat{\mathbf{a}}_{o}$ to the form $\left[\begin{array}{ll}\mathbf{a}_{o}^{t} & -1\end{array}\right]^{t}$. The final desired solution is given by $\mathbf{a}_{0}$.

Three computation issues are stated as follows: First, because QR decomposition has less computation load than SVD, QR decomposition is a better candidate when both decomposition programs are at hand. Second, unconstrained eigenfilter design needs to find the minimum eigenvector of matrix $\mathbf{Q}_{n}$ with size $(M+1) \times(M+1)$. However, new constrained eigenfilter design only requires to find the minimum eigenvector of matrix $\mathbf{B}^{t} \mathbf{Q}_{n} \mathbf{B}$ with size $(M+1-L) \times(M+1-L)$. Thus, constrained eigenfilter has less computation load in searching minimum eigenvector. Third, for eigen-approach, we are interested in only the minimum eigenvector of symmetric matrix, this computation can be performed efficiently by the conjugate gradient method [15], or iterative power method [16].

Example 2: Notch Filter Design: In this example, we will use notch filter design to demonstrate the effectiveness of proposed design algorithm described in the above. The frequency response of an ideal notch filter has unit gain for all frequency except notch frequency in which gain is zero [17]. A typical application of notch filter is to remove the $60-\mathrm{Hz}$ interference in the recording of ECG signal. Here, we will employ a case-one FIR filter of even order $N$ to design the notch filter. Thus, the 




(a)

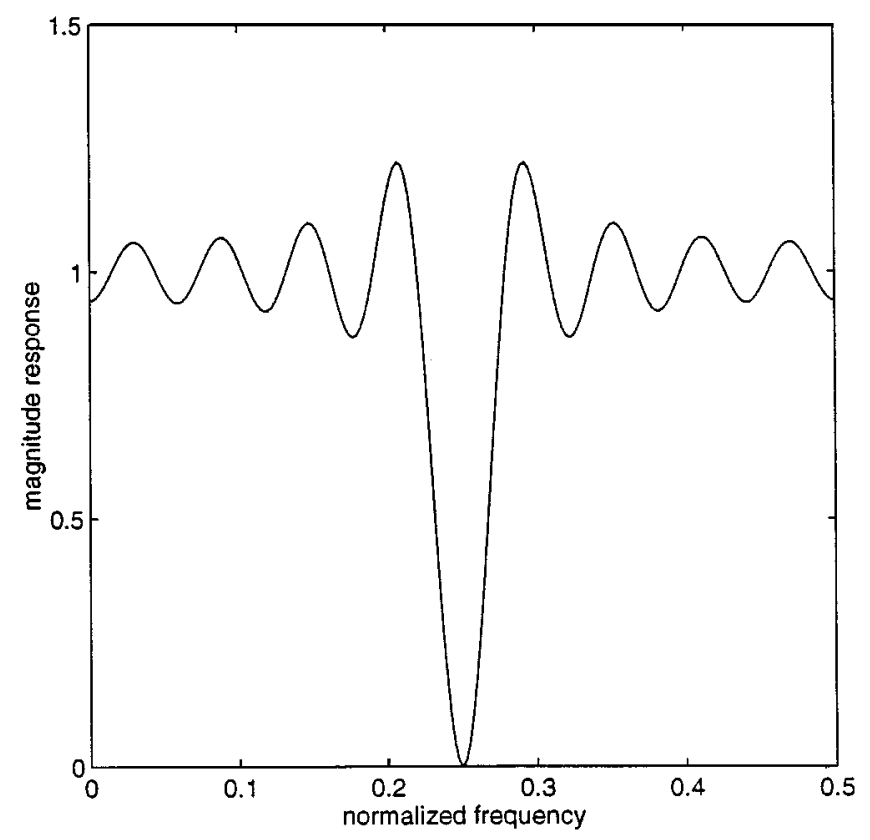

(b)

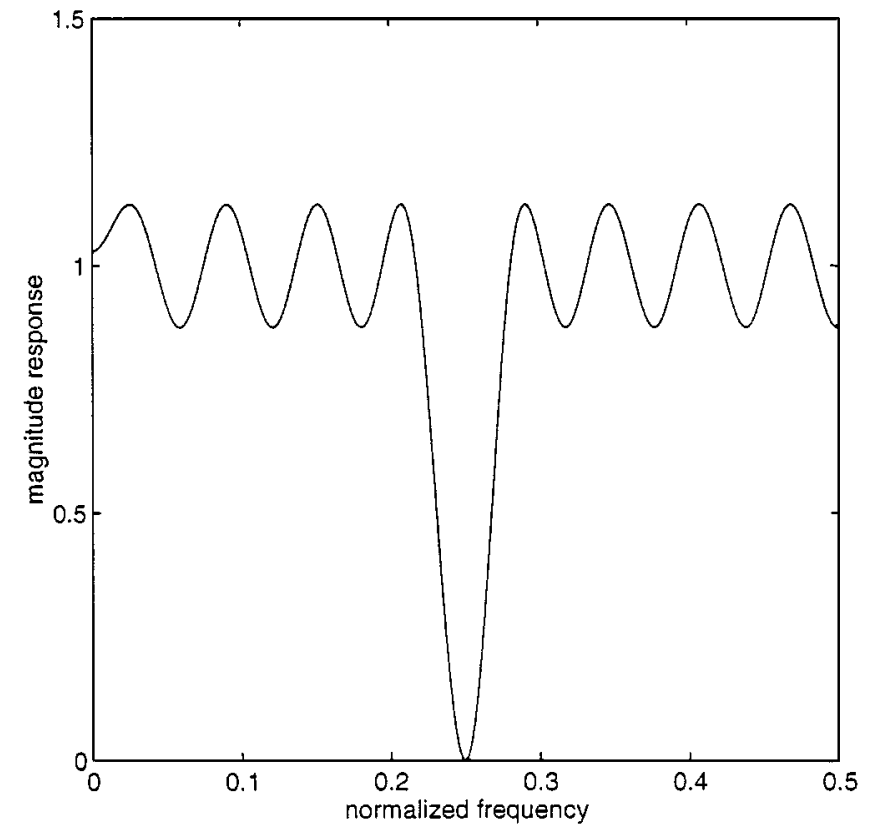

(c)

Fig. 3. (a) The magnitude response of a notch filter using new eigenfilter method. $L=1$ (solid line), $L=3$ (dashed line), $L=5$ (dotted line). (b) The magnitude response of a notch filter using Lagrange multiplier method for $L=1$. (c) The magnitude response of a notch filter using linear programming method for $L=1$.

relation of the parameter in (2) is $M=(N / 2)+1$, trig $(\omega, n)=$ $\cos ((n-1) \omega), a_{1}=h(N / 2)$, and

$a_{n}=2 h\left(\frac{N}{2}-n+1\right), \quad$ for $n=2,3, \ldots, \frac{N}{2}+1$.

Now, the optimal filter coefficient $a_{i}$ can be chosen such that the amplitude response $A(\omega)$ is as close as desired response $D(\omega)$ defined by

$$
D(\omega)=1, \quad \text { for all } \omega \in[0, \pi)
$$

Moreover, to obtain zero gain at notch frequency $\omega_{n}$ and control the null width, the following constraints are introduced:

$$
\begin{aligned}
A\left(\omega_{n}\right)= & 0 \quad \frac{\partial^{i} A\left(\omega_{n}\right)}{\partial \omega^{i}}=0, \\
& \text { for } i=1, \ldots, L-1 .
\end{aligned}
$$

After some manipulation, it can be shown that the linear constraints can be written in the standard form

$$
\mathbf{E a}=0 .
$$

Note that $\mathbf{E}$ is a $L \times M$ matrix given by

$$
\mathbf{E}^{t}=\left[\mathbf{c}_{0}^{t}, \mathbf{c}_{1}^{t}, \ldots, \mathbf{c}_{L-1}^{t}\right]
$$


where vectors

$$
\begin{aligned}
& \mathbf{c}_{0}= {\left[\begin{array}{llll}
\operatorname{trig}\left(\omega_{n}, 1\right) & \operatorname{trig}\left(\omega_{n}, 2\right) & \ldots & \operatorname{trig}\left(\omega_{n}, M\right)
\end{array}\right] } \\
& \mathbf{c}_{i}=\left[\begin{array}{lll}
\frac{\partial^{i} \operatorname{trig}\left(\omega_{n}, 1\right)}{\partial \omega^{i}} & \frac{\partial^{i} \operatorname{trig}\left(\omega_{n}, 2\right)}{\partial \omega^{i}} & \ldots \\
\frac{\partial^{i} \operatorname{trig}\left(\omega_{n}, M\right)}{\partial \omega^{i}}
\end{array}\right] .
\end{aligned}
$$

So far, the linear constraints of notch filter design has been formulated as standard form. Now, let us see some numerical examples. Fig. 3(a) shows the magnitude response of a notch filter using proposed method for $\omega_{n}=0.5 \pi, N=32$, and various $L$. It is clear that the frequency response is satisfactory. When the number of constraints $L$ increases, the notch width increases accordingly. For comparison, Fig. 3(b) and (c) show the magnitude responses of a notch filter using the well-known Lagrange multiplier and linear programming methods for $N=32, \omega_{n}=$ $0.5 \pi$, and $L=1$. Basically, the linear programming method is a minimax design and Lagrange multiplier approach is a conventional least squares design. From this result, it is obvious that proposed method almost has the same frequency response as Lagrange multiplier method. This is due to the fact that both of these methods are based on the least squares criterion. Compared with equiripple design using linear programming method, the new eigenfilter method has better frequency response fitting for all frequency band except at the range around the notch frequency $\omega_{n}$.

\section{A NeW EQUiRIPPLE EIGENFILTER DESIGN}

In the literature, it has been reported that the weighted least squares technique will produce an equiripple design if a suitable least squares frequency response weighting function is used [19]-[21]. For the conventional least squares filter design, several novel iterative algorithms for deriving the weighting function have been developed such as Lawson's algorithm and Lim's method [19]. On the other hand, the amplitude response of conventional eigenfilter can be made approximately equiripple by employing the similar iterative techniques [5]. In this section, we will present an approach to design equiripple eigenfilter based on weighted total least squares error criterion. From (17), the weighted total least squares error can be written as

$$
\begin{aligned}
J(\mathbf{a}) & =\int_{\omega \in R} W(\omega)|e(\omega)|^{2} d \omega \\
& =\int_{\omega \in R} W(\omega) \frac{\left(D(\omega)-\mathbf{a}^{t} \mathbf{c}(\omega)\right)^{2}}{\mathbf{a}^{t} \mathbf{a}+1} d \omega \\
& =\frac{\hat{\mathbf{a}}^{t} \mathbf{Q}_{w} \hat{\mathbf{a}}}{\hat{\mathbf{a}}^{t} \hat{\mathbf{a}}}
\end{aligned}
$$

where $\hat{\mathbf{a}}$ and $\mathbf{Q}_{w}$ are given by

$$
\begin{aligned}
\hat{\mathbf{a}} & =\left[\begin{array}{ll}
\mathbf{a}^{t} & -1
\end{array}\right]^{t} \\
\hat{\mathbf{c}}(\omega) & =\left[\begin{array}{ll}
\mathbf{c}(\omega)^{t} & D(\omega)
\end{array}\right]^{t} \\
\mathbf{Q}_{w} & =\int_{\omega \in R} W(\omega) \hat{\mathbf{c}}(\omega) \hat{\mathbf{c}}(\omega)^{t} d \omega .
\end{aligned}
$$

Based on Rayleigh's principle, we find the minimum eigenvector $\hat{\mathbf{a}}_{o}$ of the matrix $\mathbf{Q}_{w}$ and scale it to the form $\left[\mathbf{a}_{o}^{t}-1\right]$, then $\mathbf{a}_{o}$ is the desired optimum solution. Although optimum solution can be obtained for any given least squares weighting function, there is no known analytical method for deriving the weighting function $W(\omega)$ which will produce an equiripple design. Hence, an iterative procedure is adopted. If $W_{k}(\omega)$ is the weighting function used in the $k$ th iteration, then the weighting function $W_{k+1}(\omega)$ used in the $(k+1)$ th iteration may be expressed as

$$
W_{k+1}(\omega)=W_{k}(\omega) \beta_{k}(\omega)
$$

where $\beta_{k}(\omega)$ is given by

$$
\beta_{k}(\omega)=\left[\operatorname{env}\left(\left|D(\omega)-A_{k}(\omega)\right|\right)\right]^{\theta} .
$$

The $A_{k}(\omega)$ is the amplitude response at $k$ th iteration, env $($. is the envelope of the argument function which is formed by joining together all the extremal points of the error function $\left|D(\omega)-A_{k}(\omega)\right|$ using straight lines (see [19, Fig. 3]), and the parameter $\theta$ affects the convergence and convergent speed [19]. Once the errors become equiripple, the weighting function does not alter anymore with further iteration. If we define $q_{\max }=\max \left\{\beta_{k}(\omega)\right\}$ and $q_{\min }=\min \left\{\beta_{k}(\omega)\right\}$, then the stop criterion is given by

$$
\frac{q_{\max }-q_{\min }}{q_{\max }} \leq \varepsilon
$$

where $\varepsilon$ is a small positive number (say 0.02). Finally, the overall design procedure can be summarized as follows:

Step 1: Specify the initial weighting function as uniform weighting, i.e.,

$$
W_{0}(\omega)=1 \quad \text { for } \omega \in R \text {. }
$$

Step 2: Use (35) to obtain optimal solution of filter coefficients a and calculate initial amplitude response $A_{0}(\omega)$ and function $\beta_{0}(\omega)$. Let $k=0$.

Step 3: Use (36), (37) to compute new weighting function $W_{k+1}(\omega)$.

Step 4: Use (35) to obtain optimal solution of filter coefficients a and calculate new amplitude response $A_{k+1}(\omega)$ and function $\beta_{k+1}(\omega)$.

Step 5: Use (38) to check whether the errors are equiripple. If equiripple design is achieved, then stop iteration. Otherwise, let $k=k+1$ and go to Step 3 .

Example 3: Equiripple Lowpass Filter Design: In this example, we consider the equiripple lowpass filter design whose desired amplitude response is specified in (25) with $\omega_{p}=0.45 \pi$, and $\omega_{s}=0.55 \pi$. And, we employ the case-one FIR filter of even order $N=32$, to design this filter. The design parameters are chosen as $\varepsilon=0.02$ and $\theta=1.5$. The resultant amplitude response after eight iterations is shown in Fig. 4(a). The peak ripple magnitude of the result is 0.0213 . For comparison, the designed result using the MPR program, is also depicted in Fig. 4(b) with peak ripple magnitude 0.0213. It is clear that our approach is comparable to the MPR method. Moreover, the weighted total least squares eigenfilter can be easily modified to 
design equiripple FIR filters with arbitrary complex frequency response and in the discrete coefficient space.

\section{New Two Dimensional EIGENFILTER DESIGN}

As conventional eigenfilter, the proposed new eigen-approach is easily modified to design two-dimensional (2-D) quadrantally symmetric FIR filters [22]. The 2-D linear phase FIR digital filter with transfer function $H\left(z_{1}, z_{2}\right)$ has a frequency response

$$
\begin{aligned}
H\left(e^{j \omega_{1}}, e^{j \omega_{2}}\right)= & e^{-j N_{1} \omega_{1}} e^{-j N_{2} \omega_{2}} \sum_{m=-N_{1}}^{N_{1}} \\
& \cdot \sum_{n=-N_{2}}^{N_{2}} h(m, n) e^{-j\left(m \omega_{1}+n \omega_{2}\right)}
\end{aligned}
$$

where the impulse response $h(m, n)$ satisfies the half-plane symmetric condition, i.e.,

$$
h(m, n)=h(-m,-n)
$$

and the filter length is $\left(2 N_{1}+1\right) \times\left(2 N_{2}+1\right)$. After some manipulation, it is easily shown that the magnitude response $G\left(\omega_{1}, \omega_{2}\right)$ of $H\left(z_{1}, z_{2}\right)$ is given by

$$
G\left(\omega_{1}, \omega_{2}\right)=\sum_{i=1}^{M} a_{i} g_{i}\left(\omega_{1}, \omega_{2}\right) .
$$

The dimension $M=\left(N_{1}+1\right)+N_{2}\left(2 N_{1}+1\right)$ and

$$
\begin{aligned}
g_{i}\left(\omega_{1}, \omega_{2}\right) & =\cos \left(m \omega_{1}+n \omega_{2}\right) \\
a_{i} & = \begin{cases}h(0,0), & i=1 \\
2 h(m, n), & i \neq 1\end{cases}
\end{aligned}
$$

where relation between index $i$ and $m, n$ are given by

$$
i= \begin{cases}m+1, & \text { if } n=0 \\ & \text { and } 0 \leq m \leq N_{1} \\ \left(N_{1}+1\right)+(n-1) & \\ \left(2 N_{1}+1\right)+\left(m+N_{1}+1\right), & \text { if } 1 \leq n \leq N_{2} \\ & \text { and }-N_{1} \leq m \leq N_{1} .\end{cases}
$$

For the sake of convenience, we rewrite $G\left(\omega_{1}, \omega_{2}\right)$ in the following vector form

$$
G\left(\omega_{1}, \omega_{2}\right)=\mathbf{a}^{t} \mathbf{c}\left(\omega_{1}, \omega_{2}\right)
$$

in which the vectors are

$$
\begin{aligned}
\mathbf{a} & =\left[\begin{array}{llll}
a_{1} & a_{2} & \ldots & a_{M}
\end{array}\right]^{t} \\
\mathbf{c}\left(\omega_{1}, \omega_{2}\right) & =\left[\begin{array}{lllll}
g_{1}\left(\omega_{1}, \omega_{2}\right) & g_{2}\left(\omega_{1}, \omega_{2}\right) & \ldots & g_{M}\left(\omega_{1}, \omega_{2}\right)
\end{array}\right]^{t} .
\end{aligned}
$$

Now, the optimal filter coefficients $a_{i}$ are obtained by minimizing the total least squares error between the magnitude response $G\left(\omega_{1}, \omega_{2}\right)$ and the desired response $D\left(\omega_{1}, \omega_{2}\right)$, i.e.,

$$
\begin{aligned}
J(\mathbf{a}) & =\int \frac{\left(D\left(\omega_{1}, \omega_{2}\right)-\mathbf{a}^{t} \mathbf{c}\left(\omega_{1}, \omega_{2}\right)\right)^{2}}{\mathbf{a}^{t} \mathbf{a}+1} d \omega_{1} d \omega_{2} \\
& =\frac{\hat{\mathbf{a}}^{t} \mathbf{Q}_{2} \hat{\mathbf{a}}}{\hat{\mathbf{a}}^{t} \hat{\mathbf{a}}}
\end{aligned}
$$

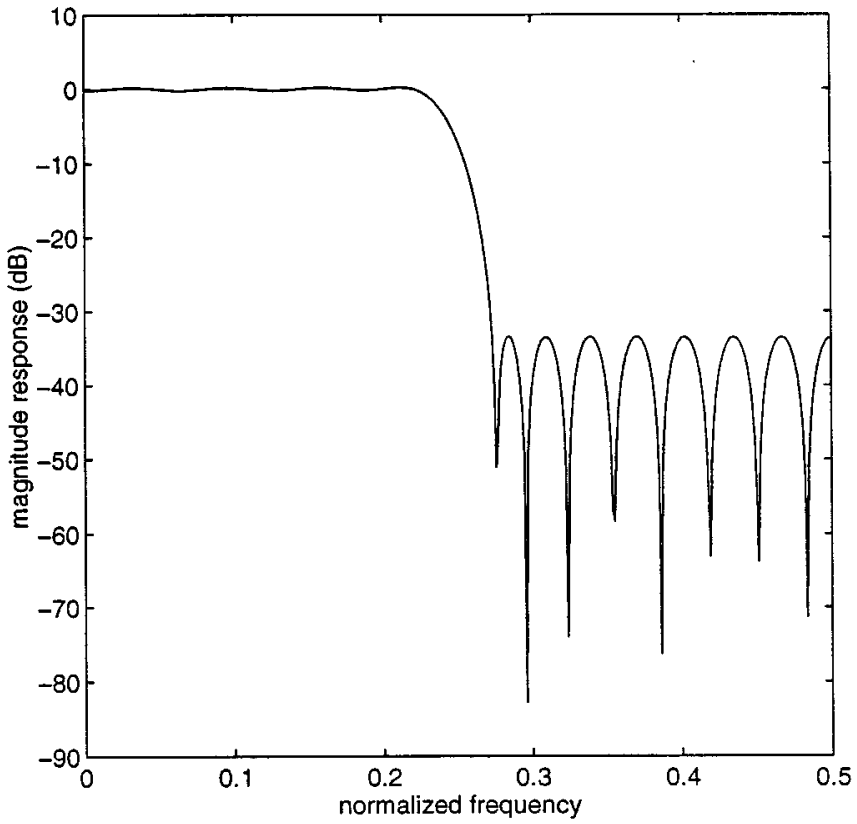

(a)

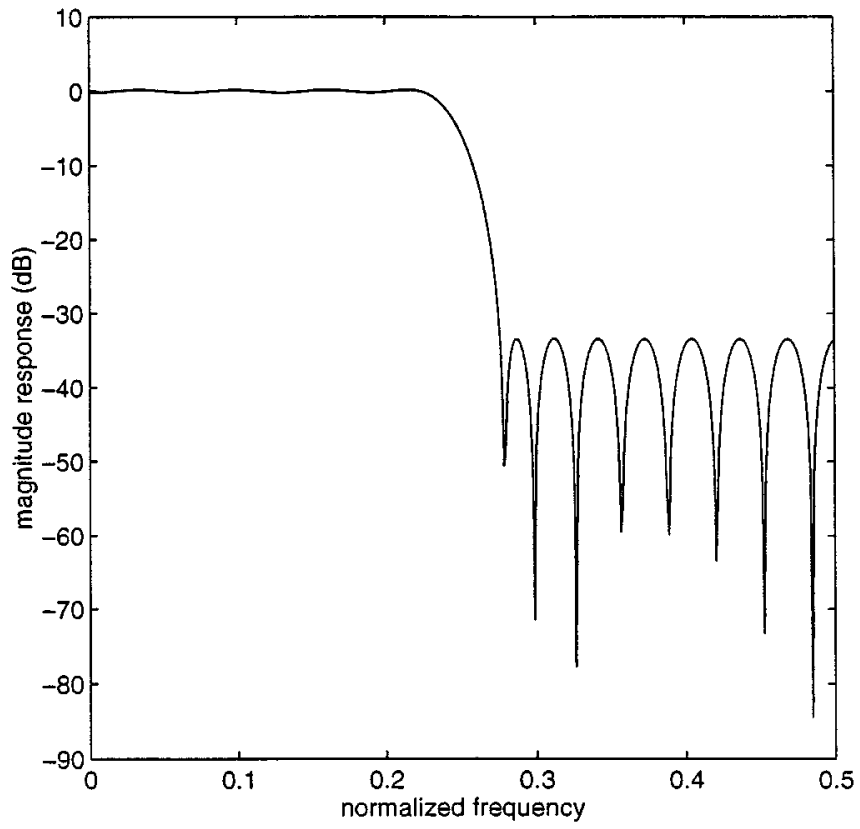

(b)

Fig. 4. (a) The magnitude response of a lowpass filter using weighted total least squares method. (b) The magnitude response of a lowpass filter using the MPR program.

where $\hat{\mathbf{a}}$ and $\mathbf{Q}_{2}$ are given by

$$
\begin{aligned}
\hat{\mathbf{a}} & =\left[\begin{array}{ll}
\mathbf{a}^{t} & -1
\end{array}\right]^{t} \\
\hat{\mathbf{c}}\left(\omega_{1}, \omega_{2}\right) & =\left[\begin{array}{ll}
\mathbf{c}\left(\omega_{1}, \omega_{2}\right)^{t} & D\left(\omega_{1}, \omega_{2}\right)
\end{array}\right]^{t} \\
\mathbf{Q}_{2} & =\int \hat{\mathbf{c}}\left(\omega_{1}, \omega_{2}\right) \hat{\mathbf{c}}\left(\omega_{1}, \omega_{2}\right)^{t} d \omega_{1} d \omega_{2} .
\end{aligned}
$$

Based on Rayleigh's principle, the minimum of $J(\mathbf{a})$ occurs at the eigenvector of the matrix $\mathbf{Q}_{2}$ corresponding to the minimum eigenvalue. The matrix $\mathbf{Q}_{2}$ is also real, symmetric and positive definite. All computation issue of the new 2-D eigenfilter 


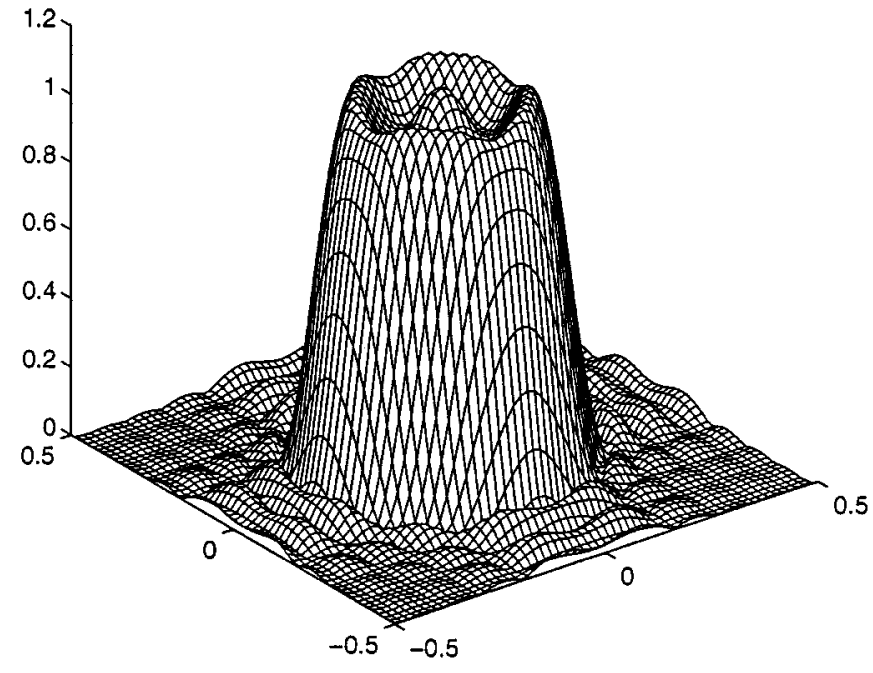

(a)



(b)

Fig. 5. (a) The magnitude response of a 2-D lowpass filter using new eigenfilter method. (b) The magnitude response of a 2-D lowpass filter using conventional eigenfilter method.

is same as the 1-D case described in the above. And, this 2-D eigenfilter is also optimal in total least squares error sense.

Example 4: 2-D Lowpass Filter Design: In this example, a 2-D circularly symmetric lowpass FIR filter of length $(11,11)$ is designed using total least squares method. The desired amplitude response is specified by

$$
D\left(\omega_{1}, \omega_{2}\right)= \begin{cases}1, & \sqrt{\omega_{1}^{2}+\omega_{2}^{2}} \leq 0.45 \pi \\ 0, & \sqrt{\omega_{1}^{2}+\omega_{2}^{2}} \geq 0.55 \pi \\ \text { don't care, } & \text { otherwise }\end{cases}
$$

The resultant magnitude response is shown in Fig. 5(a). The maximum ripple magnitudes in the passband and stopband are 0.1041 and 0.2253 , respectively. For comparison, the design result of the conventional 2-D eigenfilter approach is also depicted in Fig. 5(b) [22]. The reference point chosen is zero frequency $(0,0)$. Its maximum ripple magnitudes in the passband and stopband are 0.1081 and 0.2353 . Thus, the new 2-D eigenfilter is slightly better than the conventional 2-D eigenfilter. This is owing that the amplitude response at the reference point must be satisfied exactly for conventional 2-D eigenfilter method, but new eigenfilter is not imposed on this constraint.

\section{CONCLUSION}

In this paper, linear-phase FIR filter designs using the total least squares error criterion have been investigated. The filter coefficients are obtained from the elements of the eigenvector corresponding to minimum eigenvalue of a real, symmetric and positive definite matrix. This design is not only optimal in total least squares error sense, it is also easy to incorporate with linear constraints in time and frequency domain. Moreover, the approximation errors can be made equiripple by using iterative weighted total least squares method, and the new eigenfilter approach is easily modified to design 2 -D linear phase filters. Several design examples have been used to illustrate the effectiveness of this new design approach. However, only linear phase FIR filters are considered here. Thus, it is interesting to study IIR filter designs based on total least squares error criterion. This topic will be investigated in the future.

\section{REFERENCES}

[1] J. H. McClellan, T. W. Parks, and L. R. Rabiner, "A computer program for designing optimum FIR linear phase digital filters," IEEE Trans. Audio Electroacoust., vol. AU-21, pp. 506-526, Dec. 1973.

[2] L. R. Rabiner and B. Gold, Theory and Application of Digital Signal Processing. Englewood Cliffs, NJ: Prentice-Hall, 1975.

[3] D. W. Tufts and J. T. Francis, "Designing digital lowpass filters: Comparison of some methods and criteria," IEEE Trans. Audio Electroacoust., vol. AU-18, pp. 487-494, Dec. 1970.

[4] C. S. Burrus, A. W. Soewito, and R. A. Gopinath, "Least squared error FIR filter design with transition bands," IEEE Trans. Signal Processing, vol. 40, pp. 1327-1340, June 1992.

[5] P. P. Vaidyanathan and T. Q. Nguyen, "Eigenfilter: A new approach to least-squares FIR filter design and applications including Nyquist filters," IEEE Trans. Circuits Syst., vol. CAS-34, pp. 11-23, Jan. 1987.

[6] S. C. Pei and J. J. Shyu, "Design of FIR Hilbert transformers and differentiators by eigenfilters," IEEE Trans. Circuits Syst., vol. 35, pp. 1457-1461, Nov. 1988.

[7] — "Eigenfilter design of higher order digital differentiators," IEEE Trans. Acoust., Speech, Signal Processing, vol. 37, pp. 505-511, Apr. 1989.

[8] T. Q. Nguyen, "The design of arbitrary FIR digital filters using the eigenfilter method," IEEE Trans. Signal Processing, vol. 41, pp. 1128-1139, Mar. 1993.

[9] P. P. Vaidyanathan, Multirate Systems and Filter Banks. Englewood Cliffs, NJ: Prentice-Hall, 1993.

[10] S. C. Pei and J. J. Shyu, "Design of two-dimensional FIR eigenfilters for sampling-structure conversion," IEEE Trans. Circuits Syst. Video Technol., vol. 3, pp. 158-162, Apr. 1993.

[11] S. V. Huffel and J. Vandewalle, The Total Least Squares Problem: Computational Aspects and Analysis. Philadelphia, PA: SIAM, 1991.

[12] S. V. Huffel, Recent Advances in Total Least Squares Techniques and Errors-in-Variables Modeling. Philadelphia, PA: SIAM, 1997.

[13] D. Z. Feng, Z. Bao, and L. C. Jiao, "Total least mean squares algorithm," IEEE Trans. Signal Processing, vol. 46, pp. 2122-2130, Aug. 1998.

[14] G. Strang, Linear Algebra and Its Applications. New York: Academic, 1980 .

[15] X. Yang, T. K. Sarkar, and E. Arvas, "A survey of conjugate gradient algorithms for solution of extreme eigen-problems of a symmetric matrix," IEEE Trans. Acoust., Speech, Signal Processing, vol. 37, pp. 1550-1556, Oct. 1989.

[16] E. H. Golub and C. Van Loan, Matrix Computations. Baltimore, MD: The Johns Hopkins Univ. Press, 1983.

[17] M. H. Er, "Designing notch filter with controlled null width," Signal Processing, vol. 24, pp. 319-329, Sept. 1991.

[18] T. Chen, "Unified eigenfilter approach: With applications to spectral/spatial filtering," in Proc. IEEE Int. Symp. Circuits and Sysems, ISCAS'93, May 1993, pp. 331-334. 
[19] Y. C. Lim, J. H. Lee, C. K. Chen, and R. H. Yang, "A weighted least squares algorithm for quasiequiripple FIR and IIR digital filter designs," IEEE Trans. Signal Processing, vol. 40, pp. 551-558, Mar. 1992.

[20] S. Sunder and V. Ramachundran, "Design of equiripple nonrecursive digital differentiators and Hilbert transformers using a weighted least squares technique," IEEE Trans. Signal Processing, vol. 42, pp. 2504-2509, Sept. 1994.

[21] T. Q. Nguyen, T. I. Laakso, and R. D. Koilpillai, "Eigenfilter approach for the design of allpass filters approximating a given phase response," IEEE Trans. Signal Processing, vol. 42, pp. 2257-2263, Sept. 1994.

[22] S. C. Pei and J. J. Shyu, "2-D FIR eigenfilters: A least squares approach," IEEE Trans. Circuits Syst., vol. 37, pp. 24-34, Jan. 1990.

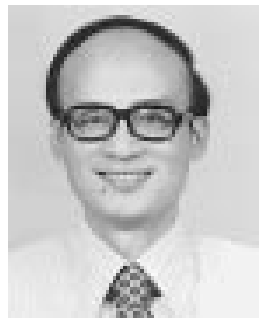

Soo-Chang Pei (S'71-M'86-SM'89-F'00) was born in Soo-Auo, Taiwan, R.O.C., in 1949. He received B.S.E.E. from National Taiwan University, Taipei, Taiwan, in 1970 and M.S.E.E. and Ph.D. degrees from the University of California, Santa Barbara in 1972 and 1975, respectively.

He was an Engineering Officer in the Chinese Navy Shipyard from 1970 to 1971 . From 1971 to 1975, he was a Research Assistant at the University of California, Santa Barbara. He was the Professor and Chairman in the EE Department of Tatung Institute of Technology, Taipei, Taiwan, and the National Taiwan University, from 1981 to 1983 , and 1995 to 1998 , respectively. He is currently a Professor in the EE Department at National Taiwan University. His research interests include digital signal processing, image processing, optical information processing, and laser holography.

Dr. Pei received the National Sun Yet-Sen Academic Achievement Award in Engineering in 1984, the Distinguished Research Award from the National Science Council from 1990 to 1998, the Outstanding Electrical Engineering Professor Award from the Chinese Institute of Electrical Engineering in 1998, and the Academic Achievement Award in Engineering from the Ministry of Education in 1998. He has been the President of the Chinese Image Processing and Pattern Recognition Society in Taiwan from 1996-1998, and is a member of Eta Kappa $\mathrm{Nu}$ and the Optical Society of America.

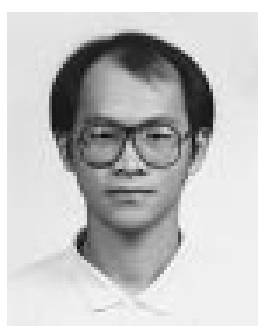

Chien-Cheng Tseng (''90-M'95) was born in Taipei, Taiwan, R.O.C., in 1965. He received the B.S. degree, (honors), from the Tatung Institute of Technology, Taipei, Taiwan, R.O.C., in 1988, and the M.S. and Ph.D. degrees from the National Taiwan University, Taipei, Taiwan, R.O.C., in 1990 and 1995, respectively, all in electrical engineering.

From 1995 to 1997, he was an Associate Research Engineer at Telecommunication Laboratories, Chunghwa Telecom Company, Ltd., Taoyuan, Taiwan, R.O.C.. He is currently an Associate Professor in the Department of Computer and Communication Engineering, National Kaohsiung First University of Science and Technology, Kaohsiung, Taiwan. His research interests include digital signal processing, pattern recognition, and electronic commerce. 